\title{
Role of Human Umbilical Cord Mesenchymal Progenitors Conditioned Media in Neuronal/Glial Cell Densities, Viability, and Proliferation
}

\author{
Antonio J. Salgado, ${ }^{1, \star}$ Joana S. Fraga, ${ }^{1, *}$ Ana R. Mesquita, ${ }^{1}$ Nuno M. Neves, ${ }^{2,3}$ \\ Rui L. Reis, ${ }^{2,3}$ and Nuno Sousa ${ }^{1}$
}

It has been recently reported that mesenchymal progenitor/stem cells isolated from the Wharton's Jelly (WJ) of umbilical cords (UC) ameliorate the condition of animals suffering from central nervous system (CNS)-related conditions. However, little is known on the mechanisms that regulate these actions. Therefore, the objective of the present work was to determine how the conditioned media (CM) of a population of mesenchymal progenitors present in the UC WJ, known as human umbilical cord perivascular cells (HUCPVCs), regulate processes such as cell viability, survival, and proliferation of postnatal hippocampal neurons and glial cells. For this purpose primary hippocampal and cortical cultures of neurons and glial cells, respectively, were incubated with CM from HUCPVCs. Results revealed that HUCPVCs CM increase glial cell viability and proliferation. Furthermore, it was observed that glial cell cultures exhibited higher numbers of GFAP-positive cells (astrocytes) and O4-positive cells (oligodendrocytes) when incubated with the CM. Additionally, it was also observed that the growth factors presents in the CM did not induce an increase on the microglial cells number. For hippocampal neurons similar results were obtained, as cultures exposed to HUCPVCs CM disclosed higher numbers of MAP-2-positive cells. Moreover it was also observed that the cell viability and proliferation in this primary hippocampal cell culture system was also higher, when compared to control cultures. From these results it was possible to conclude that HUCPVCs release neuroregulatory factors that have a direct impact on the densities, viability, and proliferation of glial cells and hippocampal primary cultures.

\section{Introduction}

$\mathrm{N}$ EUROLOGICAL DISORDERS ARE TYPICALly chronic, debilitating diseases that often lead to the premature death of afflicted individuals, thereby imposing a harsh emotional burden on their family members. These disorders, which include neurodegenerative diseases (Parkinson and Huntington disease), neuromuscular disorders, and metabolic genetic disorders, often pose unique challenges for treatment. This is mainly due to the low regenerative potential of the nervous tissue. In fact, unlike many other tissues, mature nerve cells lack the ability to regenerate, and neural stem cells, although present in the hippocampus and subventricular zone (SVZ) of the brain [1,2] have a limited ability to generate new functional neurons in response to injury
[3]. Therefore there has been an increasing interest throughout the years on the development of therapies that can adequately promote neuro-regeneration. For instance, several growth factors, with different degrees of success, have been explored for this purpose, including: nerve growth actor (NGF) [4], brain-derived neurotrophic factor (BDNF) [5], neurotrophin-3 (NT-3) [6], neurotrophin-4/5 (NT-4/5) [7], ciliary neurotrophic factor (CNTF) [8], fibroblast growth factor 2 (FGF-2) [9], and erythropoietin (EPO) [10].

The transplantation/injection of stem/progenitor cells has also emerged as a possible strategy for the restoration of function following neuronal loss. Studies from different authors [11-15] have assessed the impact of mesenchymal stem/progenitor cells isolated from bone marrow or adipose

\footnotetext{
${ }^{1}$ Life and Health Sciences Research Institute (ICVS), School of Health Sciences, University of Minho, Braga, Portugal.

${ }^{2} 3 B^{\prime}$ 's Research Group-Biomaterials, Biodegradables and Biomimetics, University of Minho, Headquarters of the European Institute of Excellence on Tissue Engineering and Regenerative Medicine, AvePark, Taipas, Guimarães, Portugal.

${ }^{3}$ Institute for Biotechnology and Bioengineering, PT Government Associated Lab, Guimarães, Portugal.

*These authors contributed equally to this work.
} 
tissue on central nervous system (CNS)-related disorders. Interestingly, the administration of these cells was shown to promote neuronal survival and regeneration, stimulating also the proliferation and differentiation of resident cells, thus limiting the extent of neurological injury and impairment in animal models of stroke [11,12], traumatic brain injury [13,14], demyelination [15], and spinal cord injury [16,17].

More recently the Wharton Jelly (WJ) of the umbilical cord (UC), a gelatinous connective tissue consisting of myofibroblast-like stromal cells, collagen fibers, and proteoglycans, was presented as source of stem/progenitor cells for CNS regeneration [18-21]. As shown by Karahuseyinoglu et al. [22] and reviewed by Troyer and Weiss [23], these cells are commonly isolated from 2 distinct areas of the UC namely the perivascular region and the intravascular area. When in culture they present a fibroblast like morphology, being positive for the expression of mesenchymal stem cell (MSC) like markers such as CD10, CD13, CD44, CD90, CD117, and negative for hematopoietic markers. Additionally they also display a multipotential character, being able to differentiate toward the osteogenic, chondrogenic, and adipogenic lineages [23]. Regarding possible applications of the latter to the CNS, Weiss and coworkers revealed that cells isolated from the bulk of the porcine WJ matrix, called umbilical cord matrix cells (UCMCs), could be observed in the brains of rats following transplantation without immunosuppressive therapy [18]. Subsequent studies from the same authors have demonstrated that these cell populations could have an impact on the treatment of neurodegenerative diseases, such as Parkinson's disease (PD) [19,20]. In fact, it was shown that injection of these cells in PD-induced animals triggered a behavioral improvement and increased the number of tyrosine hydroxylase (TH)-positive neurons [20]. Finally Hirko et al. [24] also demonstrated that UCSCs were able to modulate the inflammatory reaction within the brain, after global ischemia, disclosing a neuroprotector role within the CNS. However, little is known concerning the mechanisms underlying the interactions between the stem cell populations derived from the umbilical cord and the residing neuronal and glial cells. Although it has been reported that these cells differentiate toward the neuronal lineage [21,25], it is most likely that, similarly to what has been described to bone marrow mesenchymal stem cells [26-29], these effects are mediated through the release of cytokines and growth factors.

Therefore, in the present study we determined how the conditioned media (CM) of human umbilical cord perivascular cells (HUCPVCs), a population of cells with a known mesenchymal stem cell-like character [30,31], interact with primary cultures of post-natal hippocampal neurons and glial cells. As endpoints of these interactions, we assessed cell viability, proliferation, and differentiation of CNS resident cells. Results revealed that HUCPVCs CM contained growth factors that increased astrocyte, oligodendrocyte, and neuronal metabolic viability, proliferation, and cell densities.

\section{Materials and Methods}

\section{Cell culture}

Human umbilical cord perivascular cells. HUCPVCs were either a kind gift from Dr. J. E. Davies, University of Toronto, or isolated from the UCs from consenting full-term caesarean section patients. Ethical approval had been previously obtained from the University of Toronto/Sunnybrook and Women's College Health Sciences Centre, Toronto or the Hospital de S. Marcos, Braga, Portugal. HUCPVCs were isolated according to the procedure originally described by Sarugaser et al. [30]. Pieces of cord, $4-5 \mathrm{~cm}$ long, were dissected by first removing the epithelium of the UC section along its length to expose the underlying WJ. Each vessel, with its surrounding WJ matrix, was then pulled away, after which the ends of each dissected vessel were tied together with a suture creating "loops" that were placed into a $50-\mathrm{mL}$ tube containing a solution of $0.5-0.75 \mathrm{mg} / \mathrm{mL}$ collagenase (Sigma, St. Louis, MO) with phosphate-buffered saline (PBS, Invitrogen/Gibco, Carlsbad, $\mathrm{CA})$. After $18 \mathrm{~h}$, the loops were removed from the suspension, which was then diluted with PBS to reduce the viscosity of the suspension and centrifuged. Following the removal of the supernatant, cells were resuspended in culture media, $\alpha$-MEM (Invitrogen/Gibco) supplemented with 10\% FBS (Invitrogen/ Gibco) and 1\% antibiotic/antimycotic (Sigma), counted using a hemocytometer and platted in T75 flasks at a density of 4,000 cells/cm 2 . The culture medium was changed every $2 / 3$ days. Upon confluence cells were trypsinized and passaged to new T75 flasks.

Primary cultures of hippocampal neurons. Hippocampal neurons cultures were prepared from P4 Wistar Rats [32]. Briefly, and upon dissection, hippocampi were submitted to a trypsin-based enzymatic digestion followed by mechanical dissociation. Isolated cells were then plated on coverslips previously coated with poly-D-lysine (Sigma) at a density of 40,000 cells $/ \mathrm{cm}^{2}$. Characterization of the cultures by immunocytochemistry (microtubule-associated protein (MAP-2)neurons; glial acidic fibrillary protein (GFAP)-astrocytes; nestin-neuroprogenitors) revealed that they possessed approximately $45 \%-50 \%$ of mature neurons, $5 \%-10 \%$ of astrocytes, and $35 \%-40 \%$ of neural progenitors, whenever grown in Neurobasal media supplemented with B27 (Invitrogen/Gibco) and FGF-2 (Invitrogen/Gibco). In the absence of these factors, there is a marked decrease in cell viability and the cultures remain essentially in an undifferentiated state. There is also a small contamination by other cell types, namely fibroblasts and endothelial cells, which comprise $<1 \%$ of the total culture.

Primary cultures cortical glial cells. Cortical glial cells were isolated from $\mathrm{P} 4$ newborn Wistar rats. Upon dissection, cortices were enzymatically digested $(30 \mathrm{mg} / \mathrm{mL}$ DNase, $0.25 \%$ Trypsin, both from Sigma) followed by a strong mechanical dissociation. The mechanical dissociation allows a better separation of the cells from the surrounding tissue. Simultaneously leads to cell death along neuronal cell populations, which are known to be quite more sensitive to these procedures. Glial cells were then obtained by centrifuging the resulting cell suspension at $800 \mathrm{rpm}$ for $2 \mathrm{~min}$, and plated out on coverslips previously coated with poly-D-lysine at a density of 40,000 cells $/ \mathrm{cm}^{2}$. As for the hippocampal cultures an immunocharacterization was performed, showing that the dominant cell type are microglial cells $(45 \%-55 \%)$, followed by astrocytes (35\%-45\%) and oligodendrocytes $(5 \%-10 \%)$. Similarly to what happened for neuronal cultures, there is a small contamination by other cell types, namely fibroblasts, which comprise $<1 \%$ of the total culture.

\section{Conditioned medium collection and experiments}

Conditioned media (CM) were collected from P2 HUCPVCs. For this purpose cells were plated out at a 
density of 4,000 cells $/ \mathrm{cm}^{2}$ and allowed to grow for 3 days. Following this, culture medium was renewed and CM collected 24, 48, and $72 \mathrm{~h}$ thereafter (cell culture media was not renewed or added during this time period). Upon collection $\mathrm{CM}$ were frozen, being later on thawed on the day of the experiments. For CM collection standard HUCPVCs culture medium was used for experiments with glial cells, while for experiments with hippocampal neurons, Neurobasal-A medium supplemented was the chosen medium. Experiments with glial cells and hippocampal neurons were done as follows:

Hippocampal neurons. Upon isolation hippocampal neurons were plated out at the densities referred above and incubated from T0 with the previously collected CM $(n=3$ / $\mathrm{CM}$ time point) for 7 days (with half of the volume of $\mathrm{CM}$ being renewed at day 4 of culture), after which cell densities, viability, and proliferation were assessed (see below). Besides kanamycin and glutamax, no further supplements were added to the HUCPVCs CM. Control cultures were kept in Neurobasal media supplemented with kanamycin and glutamax.

Glial cells. Upon isolation as referred in 2.1.3, glial cells were allowed to grow for 5 days. On day 5 the culture medium was switched to CM ( $n=3 / \mathrm{CM}$ time point); after incubation for further 9 days cell densities, viability, and proliferation were assessed (see below). Control cultures were kept in $\alpha$-MEM supplemented with $1 \%$ antibiotics/ antimycotics (Sigma).

The different time periods of CM incubation for neurons and glial cells were mainly related to their maturation stage. For hippocampal neurons the maturation stage is 7 days while for cortical glial cells is 14 days [32,33].

\section{Cell viability assessment}

Cell viability was assessed by the MTS test. The MTS [3-(4,5-dimethylthiazol-2-yl)-5-(3-carboxymethoxyphenyl)2(4-sulfophenyl)-2H tetrazolium] (Promega, Madison, WI) test is an assay in which the substrate-MTS-is bioreduced into a brown formazan product by NADPH or NADP produced by mitochondrial enzymes, which are active in living cells $[34,35]$. It is similar to MTT test, presenting the advantage of producing a final formazan product that is soluble in aqueous media. Cell culture coverslips $(n=3)$ were placed in culture medium containing MTS in a 5:1 ratio and incubated in a humidified atmosphere at $37^{\circ} \mathrm{C}$ and $5 \% \mathrm{CO}_{2}$. After $3 \mathrm{~h}$ of incubation, $100 \mu \mathrm{L}$ of solution from each sample were transferred to 96-well plates and the optical density was determined at $(450 \mathrm{~nm})(n=3 / \mathrm{CM}$ time point $\pm S D$ ).

\section{Cell proliferation}

Cell proliferation was determined by a colorimetric assay based on 5-bromo-2'-deoxyuridine (BrdU) incorporation (Roche, Mannheim, Germany). Primary hippocampal and glial cell cultures incubated with $\mathrm{CM}$, and respective controls, were incubated with BrdU on days 6 and 13, respectively. After $24 \mathrm{~h}$ of incubation, ELISA test was performed according to the company's instruction in the end of which $\mathrm{OD}$ was determined at $450 \mathrm{~nm}$ with a reference filter at 655 $\mathrm{nm}$. Results are shown as a ratio between CM-incubated cultures and controls $(n=3 / \mathrm{CM}$ time point $\pm \mathrm{SD})$.

\section{Total protein quantification}

Total protein was quantified $(n=3 / \mathrm{CM}$ time point and control) through the bicinchoninic acid (BCA) Protein Assay kit (Pierce Chemical, USA), as previously demonstrated by Silva et al. [36]. This system uses BCA as the detection reagent for $\mathrm{Cu}^{1+}$, which is formed when $\mathrm{Cu}^{2+}$ is reduced by protein in an alkaline environment. The purple colored reaction product is formed by the chelation of 2 molecules of BCA with 1 cuprous ion $\left(\mathrm{Cu}^{1+}\right)$. At the end of the assay, $100 \mu \mathrm{L}$ of each sample were transferred to 96-well plates where a standard curve was prepared and the absorbance read at $562 \mathrm{~nm}$ in a multi-well plate reader (Tecan, Männedorf, Switzerland).

\section{Immunocytochemistry}

Cells were fixed in $4 \%$ paraformaldehyde for $30 \mathrm{~min}$, permeabilized by incubation with $0.3 \%$ Triton X-100 in PBS for 5 $\mathrm{min}$ at room temperature (for neurons and astrocytes), and washed 3 times in PBS. Cells were then blocked with $10 \%$ FBS/PBS (60 min), followed by a 60-min incubation with the primary antibodies: mouse anti-rat microtubule-associated protein 2 (MAP-2) (Sigma) to detect mature hippocampal neurons, rabbit anti-rat glial fibrillary acid protein (GFAP) (Dako, Denmark) for astrocytes, mouse anti-CD11b (BD Biosciences Pharmigen, San Diego, CA) for microglial cells, and mouse anti-O4 (R\&D systems, Minneapolis, MN) for oligodendrocytes. Cells were then washed in PBS and incubated with Alexa Fluor 594 goat anti-mouse immunoglobulin G (IgG; Invitrogen/Molecular Probes, Carlsbad, CA) or Alexa Fluor 488 rabbit anti-rat IgG (Invitrogen/Molecular Probes). Primary antibody was omitted to produce negative controls. Samples were observed under an Olympus BX-61 Fluorescence Microscope (Olympus, Germany).

\section{Cell counts}

Cell counts were performed by using Cell-P software (Olympus, Germany). For this purpose 3 coverslips per condition and 3 representative fields were chosen and analyzed. In order to normalize the data between the different sets of cell culture, results are presented in number of cells/ $\mu \mathrm{g}$ protein.

\section{Statistical analysis}

Statistical evaluation was performed using one-way ANOVA to assess the statistical differences between different groups. Statistical significance was defined as $P<0.05$ for a $95 \%$ confidence interval.

\section{Results}

In the present study we aimed at determining to what extent a population of mesenchymal progenitors derived from the umbilical cord, known as HUCPVCs, could influence the in vitro densities, viability, and proliferation of post-natal hippocampal neurons and cortical glial cell populations.

In Figure 1, it is possible to observe that the incubation of glial cells with CM from HUCPVCs lead to an overall increase of cell metabolic viability. All the values were above and significantly different $(P<0.05)$ when compared to control conditions. Moreover, it could also be observed that 
A

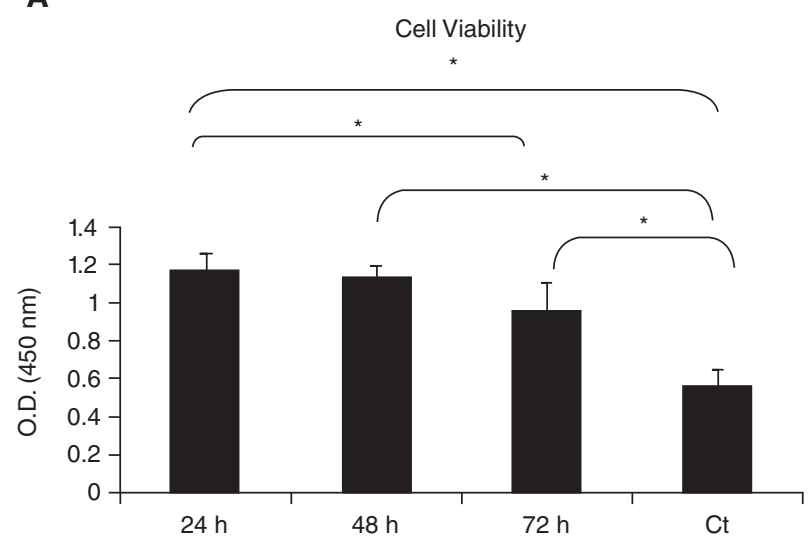

C

Astrocytes

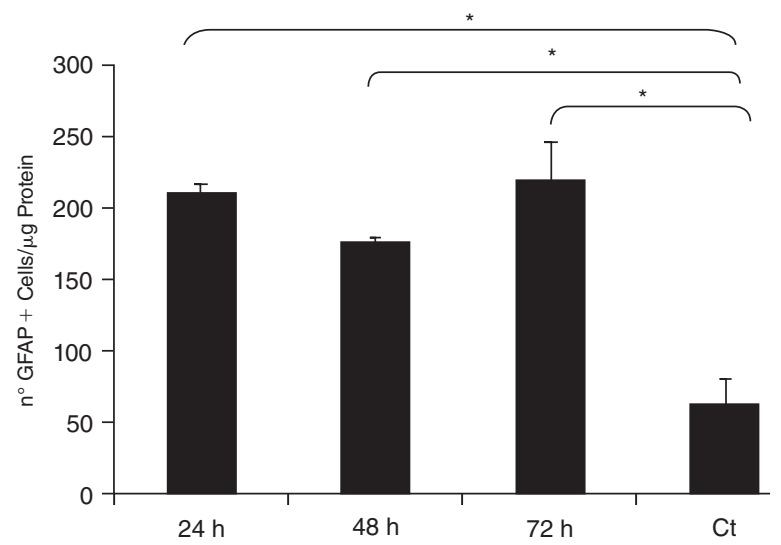

E

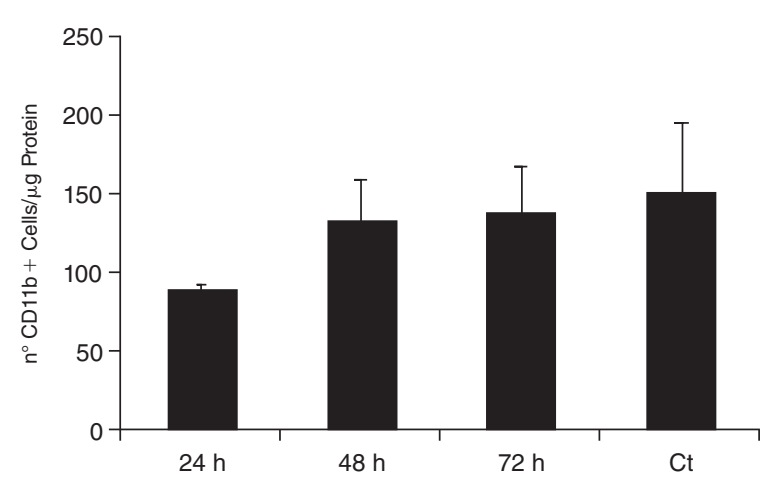

B

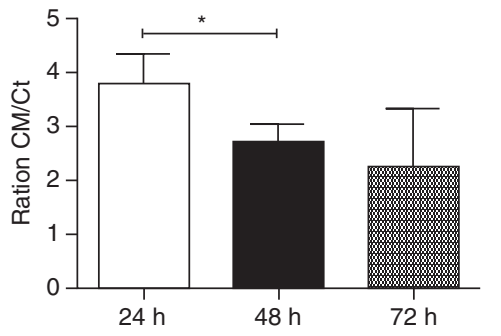

D

Oligodendrocytes

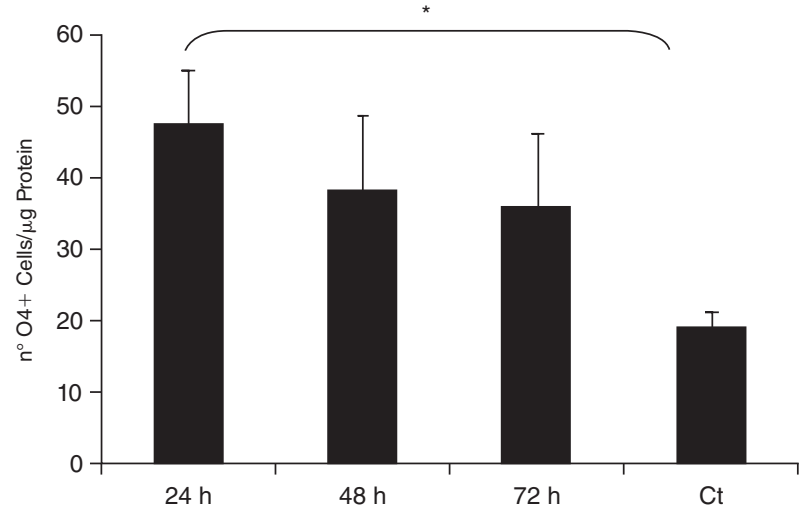

FIG. 1. Glial cell incubation with human umbilical cord perivascular cells (HUCPVCs) conditioned media (CM). Experiments revealed that HUCPVCs CM increased glial cell viability (A) and proliferation (B) (all the values were statistically significant different from the control, one way ANOVA, $n=3$, mean $\pm \mathrm{SD}, P<0.05$ ). For the immunocytochemistry glial cell cultures were stained for GFAP, Astrocytes; CD11b, Microglial cells; and O4, Oligodendrocytes. Total cell counts normalized to the content of total protein/well (C, D, E; cells/ $\mu$ g protein) revealed that these effects were mainly exerted on astrocytes (all CM time points statistically different from the control, one way ANOVA, $n=3$, mean $\pm \mathrm{SD}, P<0.05$ ) and oligodendrocytes (CM 24 h one way ANOVA, $n=3$, mean $\pm \mathrm{SD}, P<0.05$ ). Although nonsignificative (one way ANOVA, $n$ $=3 \pm \mathrm{SD}, P>0.05$ ) results obtained with microglial cells suggest that CM 24 h reduce the number of microglial cells. This fact alludes to the possible low inflammatory character of HUCPVCs.

there were also differences between the groups incubated with CM, namely between CM $24 \mathrm{~h}$ and CM $72 \mathrm{~h}(P<0.05)$. Additionally it was also possible to observe that cell proliferation, as assessed by BrdU incorporation, was up-regulated in all CM-incubated cultures. This was observed namely for CM $24 \mathrm{~h}$ displaying a nearly 2-fold increase when directly compared to control cultures. Given that the glial cell culture system used in the present work contained all the glial 
cell types (astrocytes, oligodendrocytes, and microglial cells), we then proceeded to determine the effect of the CM incubation at the subpopulation level. As it can be observed in Figures 1 and 2, HUCPVCs' CM had clear effects on the final densities of both astrocytes and oligodendrocytes. This effect was present for all CM time points $(P<0.05)$ in astrocytic populations, while for oligodendrocytes there is, apparently, a temporal profile of growth factor expression that clearly stimulates oligodendrocyte cell densities at CM $24 \mathrm{~h}$ $(P<0.05)$, starting to decay from then on. Regarding microglial cells, it was observed that cultures incubated with CM did not disclose higher values than those presented by control samples. In fact, the graph shown in Figure 1E indicates that cultures incubated with HUCPVCs CM had lower cell densities of microglial cells, namely those cultured with CM $24 \mathrm{~h}$.

Figures 3 and 4 show the results for the experiments performed with primary hippocampal cultures. Cell viability assays revealed that in the absence of supplements the control cultures disclosed lower levels of cell viability, up to 3-fold smaller $(P<0.05)$ when compared to the CM-incubated cultures (Fig. 3A). Simultaneously, it was also possible to observe that CM $24 \mathrm{~h}$ (Fig. 3B) drastically increased cell proliferation in this culture system $(P<0.05)$, while CM $48 \mathrm{~h}$ and $72 \mathrm{~h}$ did not induce any changes in these parameters. MAP-2 immunostaining showed that no differences were observed between the different CM groups for neuronal densities, but drastic differences were observed when compared to the control $(P<0.05)$, as the latter had almost undetected numbers of MAP-2-positive cells (Figs. 3C and 4).

\section{Discussion}

As one of the possible mechanisms by which non-neural adult stem cells act on neuronal cell populations is through the release of cytokines, growth factors, and neuroregulatory molecules, our work consisted on the incubation of hippocampal neurons and glial cells with CM obtained from HUCPVCs at different time points.
Our results showed that the CM, and thus the growth factors secreted by HUCPVCs, did have a positive impact on different parameters of a primary glial cell culture system. Moreover, this input of HUCPVCs CM on glial cells is mediated by proteic factors, as after heating up the different $\mathrm{CM}$ and therefore denaturing the proteic content the same effects were not observed (data not shown). With the present experiment, it was possible to observe that the secreted proteins by HUCPVCs could increase the levels of cell viability and proliferation. In both cases CM $24 \mathrm{~h}$ has a more striking effect than the other CM. Although there is not a concluding reason for this observation, it is true that some nutrient depletion may occur while CM is being collected. However, as no major differences are found between CM $48 \mathrm{~h}$ and $72 \mathrm{~h}$ it would be also reasonable to suggest that the half-life of the secreted factors could also be playing a role on this subject. This is even more evident for the cell proliferation, where a near 2-fold decrease was observed when cultures were incubated with these 2 groups of CM. At the subpopulation level, it is possible to state that HUCPVCs release factors to the extracellular milieu that have a direct impact on the densities of astrocytes and oligodendrocytes. However, the response obtained by these 2 different cell subpopulations was different. Oligodendrocytes were more sensitive to the temporal profile of CM collection. A closer observation of these results allows to establish a comparison to temporal profiles obtained for cell viability and proliferation, as all of them present the same trend. Once again this could be related to the half-life of the secreted factor(s) that is (are) playing a role on these observations. Nevertheless further studies need to be conducted in order to fully understand the reasons behind the observed phenomena. Finally, the results obtained for microglial cells suggest that the proteic factors released by the HUCPVCs did not induce the proliferation of these mediators of inflammatory response within the CNS and, therefore, alludes to the low inflammatory potential of these CM. This is would be in accordance to what has been previously described by Hirko et al. [24] and Ennis et al. [37] in mixed lymphocyte cultures.
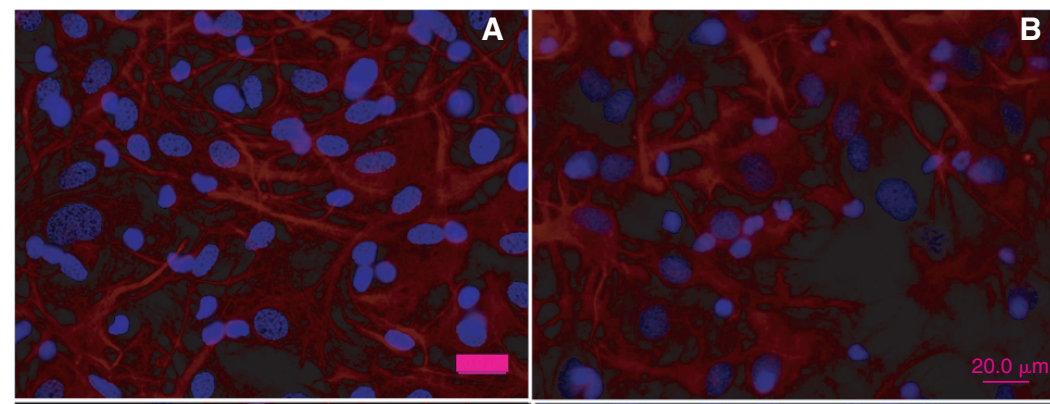

C

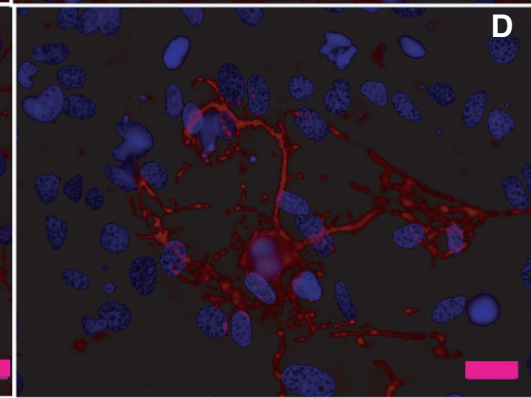

FIG. 2. Glial cell incubation with human umbilical cord perivascular cells (HUCPVCs) conditioned media (CM). Immunocytochemistry staining for GFAP (A, CM 24 h; B, Ct) and O4 (C, CM 24 h; D, Ct) revealed that HUCPVCs released proteic factors that induce an increase on astrocyte and oligodendrocyte cell densities. Abbreviations: GFAP, astrocytes; O4, Oligodendrocytes. 
A

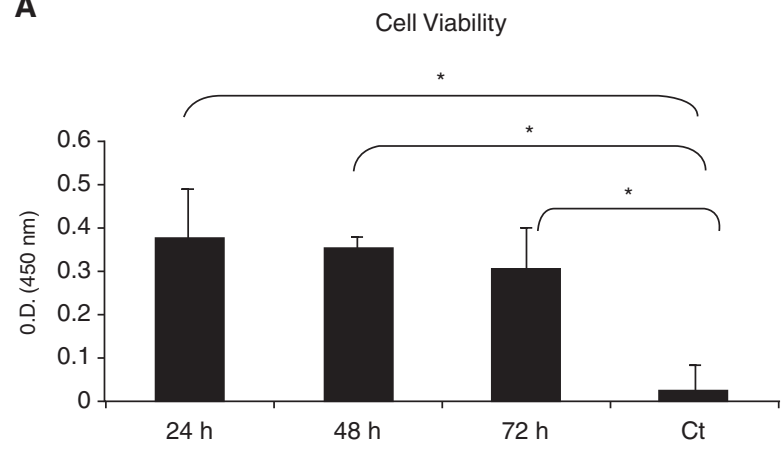

C

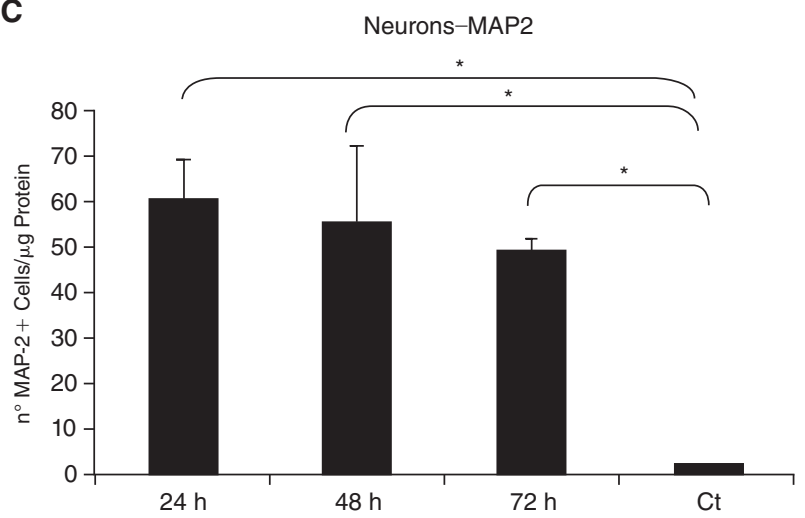

B

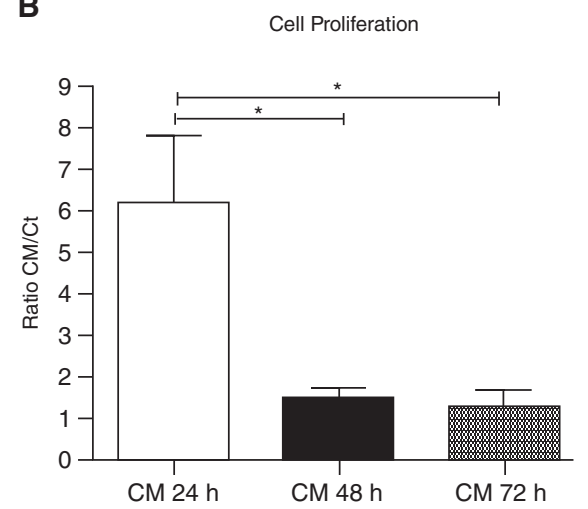

FIG. 3. Hippocampal neurons incubated with human umbilical cord perivascular cells (HUCPVCs) conditioned media (CM). Similarly to what was observed for glial cells, CM induced an increase of metabolic viability (all values statistically significant different from the control, one way ANOVA, $n=3$, mean \pm for $\mathrm{SD}, P<0.05)$ was observed. MAP-2 cell counts normalized to the content of total protein/well revealed that there were drastic differences to the control (one way ANOVA, $n=3$, mean $\pm \mathrm{SD}, P<0.05$ ). Therefore it can be stated that HUCPVCs CM possess neuroregulatory molecules that increase cell viability, proliferation and survival/differentiation on primary cultures of hippocampal neurons.

For the experiments with hippocampal neurons similar results were obtained for cell viability and proliferation. As it can be concluded from Figures 3 and 4, the CM collected from HUCPVCs for all time points increased the overall cell metabolic viability when compared to control cultures. Moreover it was also possible to observe that CM $24 \mathrm{~h}$ had notorious effects on the up-regulation of cell proliferation $(P<0.05)$. From these data one can conclude that the tested $\mathrm{CM}$ had different effects on the analyzed parameters, and thus CM $24 \mathrm{~h}$ is the most adequate to induce proliferation. Additionally immunocytochemistry for MAP-2 allowed to observe that the cultures incubated with the different $\mathrm{CM}$ had a higher number of MAP-2-positive cells when compared to controls. This result was expected from control cultures, as in the absence of neuronal supplements such as B27, the latter remain mainly in the undifferentiated state. Furthermore, it was also possible to observe that, in what concerns neuronal survival/differentiation, there are no differences in the trophic capability of HUCPVCs CM different time points of collection. These observations are a clear demonstration that HUCPVCs do in fact released neuroregulatory molecules that promote neural survival/differentiation as no neural inducing factors were added to the $\mathrm{CM}$. Moreover these factors seem to compensate the effects caused by the nutrient depletion during the $\mathrm{CM}$ collection, as otherwise cell viability and MAP-2 positive cell densities would have been decreased. Nevertheless the nature of these factors is at the moment unknown.
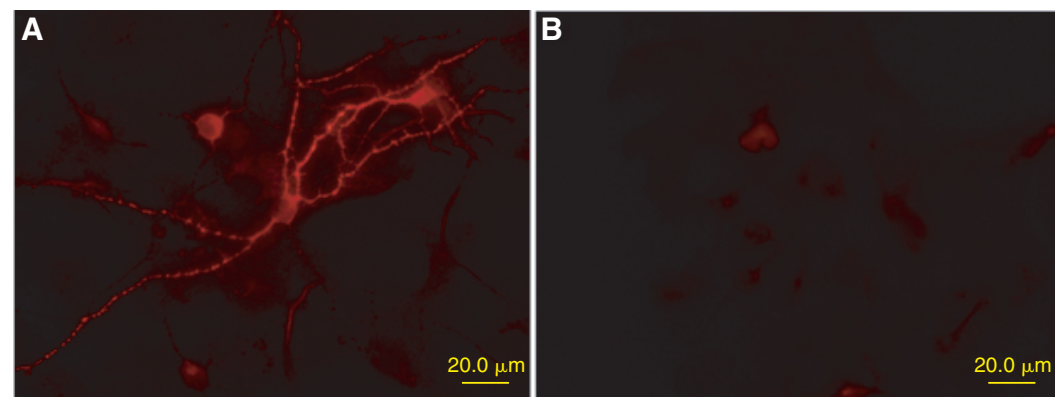

FIG. 4. Immunocytochemistry staining for microtubule-associated protein 2 (MAP-2) on hippocampal neurons incubated with human umbilical cord perivascular cells (HUCPVCs) conditioned media (CM) (A) and respective control (B). It was observed that HUCPVCs CM induced a higher degree of neuronal survival/ differentiation when compared to controls. 


\section{Conclusions}

With the present work, it was possible to demonstrate that the $\mathrm{CM}$ of a population of mesenchymal progenitors present in the UC's WJ, HUCPVCs, is able to increase cell viability and proliferation on primary cultures of glial cells. Additionally they also potentiate astrocyte and oligodendrocyte cell densities, without stimulating the proliferation of microglial cells, the immune system mediators of the CNS. Furthermore, it was also revealed that the secretome of HUCPVCs might also play a role in potentiating neural progenitors proliferation, as well as neuronal survival/differentiation. The experiments herein described, for both glial cells and hippocampal neurons, suggest that most of these effects are mediated by the release of growth/trophic factors by HUCPVCs in the extracellular milieu; future experiments will focus on the identification of these factors.

\section{Acknowledgments}

Portuguese Foundation for Science and Technology through funds from POCTI and/or FEDER programs (funding to ICVS, 3B's Research Group and post doctoral fellowship to A.J. Salgado-SFRH/BPD/17595/2004); funding from Fundação Calouste de Gulbenkian. The authors would also like to acknowledge Prof. J.E. Davies from the Institute of Biomaterials and Biomedical Engineering at the University of Toronto, Canada, for kindly providing some of the HUCPVCs lots used in the present work. This work was performed following the terms of the cooperation agreement signed between the 3B's Research Group of the University of Minho and the Hospital de São Marcos in Braga and approved by its ethical committee.

\section{Author Disclosure Statement}

No competing financial interests exist.

\section{References}

1. Morshead CM, BA Reynolds, CG Craig, MW McBurney, WA Staines, D Morassuti, Weiss S and Van der Kooy D. (1994). Neural stem cells in the adult mammalian forebrain: a relatively quiescent subpopulation of subependymal cells. Neuron 13:1071-1082.

2. Schneider A, C Krügler, T Steigleder, D Weber, C Pitzer, R Laage, J Aronowski, MH Maurer, N Gassier, W Mier, M Hasselblat, R Kollmar, S Schwab, C Sommer, A Bach, HG Kuhn and WR Schäbitz. (2005). The hematopoietic factor G-CSF is a neuronal ligand that counteracts programmed cell death and drives neurogenesis. J Clin Invest 115:2083-2098.

3. Kang SK, DH Lee, YC Bae, HK Kim, SY Baik and JS Jung. (2003). Improvement of neurological deficits by intracerebral transplantation of human adipose tissue-derived stromal cells after cerebral ischaemia in rats. Exp Neurol 183:355-366.

4. Fiore M, V Triaca, T Amendola, P Tirassa and P Aloe. (2002). Brain NGF and EGF administration improves passive avoidance response and stimulates brain precursor cells in aged male mice. Physiol Behav 77:437-443.

5. Sharfman H, J Goodman, A Macleod, S Phani, C Antonelli and $S$ Croll. (2005). Increased neurogenesis and the ectopic granule cells after intrahippocampal BDNF infusion in adult rats. Exp Neurol 192:348-356.

6. Scarisbrick LA, K Asakura and M Rodriguez. (2000). Neurotrophin-4/5 promotes proliferation of oligodendrocyte type 2 astrocytes (O-2A). Brain Res Dev Brain Res 123:87-90.
7. Collazo D, H Takahashi and RD Mckay. (1992). Cellular targets and trophic functions of neurotrophin-3 in the developing rat hippocampus. Neuron 9:643-656.

8. Emsley JG and T Hagg. (2003). Endogenous and exogenous ciliary neurotrophic factor enhances forebrain neurogenesis in adult mice. Exp Neurol 183:298-310.

9. Palmer TD, J Ray and FH Gage. (1995). FGF-2 responsive neuronal progenitors reside in proliferative and quiescent regions of the adult rodent brain. Mol Cell Neurosci 6:474-486.

10. Shingo T, ST Sorokan, T Shimazaki and S Weiss. (2001). Erythropoietin regulates the in vitro and in vivo production of neuronal progenitors by mammalian forebrain neural stem cells. J Neurosci 21:9733-9743.

11. Chen X, Y Li, L Wang, M Katakowski, L Zhang, J Chen, Y Xu, SC Gautam and M Chopp. (2002). Ischemic rat brain extracts induce human marrow stromal cell growth factor production. Neuropathology 22:275-279.

12. Zhao LR, WM Duan, M Reyes, CD Keene, CM Verfaillie and WC Low. (2002). Human bone marrow stem cells exhibit neural phenotypes and ameliorate neurological deficits after grafting into the ischemic brain of rats. Exp Neurol 174:11-20.

13. Lu D, A Mahmood, L Wang, Y Li, M Lu and M Chopp. (2001). Adult bone marrow stromal cells administered intravenously to rats after traumatic brain injury migrate into the brain and improve neurological outcome. Neuroreport 12:559-563.

14. Mahmood A, D Lu, M Lu and M Chopp. (2003). Treatment of traumatic brain injury in adult rats with intravenous administration of human bone marrow stromal cells. Neurosurgery 53:697-702.

15. Akyama Y, C Radtke and JD Kocsis. (2002). Remyelination of the rat spinal cord by transplantation of identified bone marrow stromal cells. J Neurosci 22:6623-6630.

16. Kang SK, MK Shin, JS Jung, YG Kim and CH Kim. (2006). Autologous adipose tissue-derived stromal cells for treatment of spinal cord injury. Stem Cells Dev 15:583-594.

17. Kang SK, JE Yeo, SK Kang and DG Phinney. (2007). Cytoplasmic extracts from adipose tissue stromal cells alleviates secondary damage by modulating apoptosis and promotes functional recovery following spinal cord injury. Brain Pathol 17:263-275.

18. Weiss ML, KE Mitchell, JE Hix, S Medicetty, SZ El-Zarkouny, D Grieger and DL Troyer. (2003). Transplantation of porcine umbilical cord matrix cells into the rat brain. Exp Neurol 182:288-299.

19. Medicetty S, AR Bledsoe, CB Fahrenholtz, D Troyer and ML Weiss. (2004). Transplantation of pig stem cells into rat brain: proliferation during the first 8 weeks. Exp Neurol 190:32-41.

20. Weiss ML, S Medicetty, R Bledsoe, RS Rachakatla, M Choi, S Merchay, Y Luo, MS Rao, G Velagaleti and D Troyer. (2006). Human umbilical cord matrix stem cells: preliminary characterization and effect of transplantation in a rodent model of Parkinson's Disease. Stem Cells 24:781-792.

21. Fu YS, YC Cheng, MY Lin, H Cheng, PM Chu, SC Chou, YH Shih, MH Ko and MS Sun. (2006). Conversion of human umbilical cord mesenchymal stem cells in Wharton's Jelly to dopaminergic neurons in vitro: potential therapeutic application for Parkinsonism. Stem Cells 24:115-124.

22. Karahuseyinoglu S, O Cinar, E Kilic, F Kara, GG Akay, DO Demiralp, A Tukun, D Uckan and A Can. (2007). Biology of stem cells in human umbilical cord stroma: in situ and in vitro surveys. Stem Cells 25:319-331.

23. Troyer DL and ML Weiss. (2008). Wharton's jelly-derived cells are a primitive stromal cell population. Stem Cells 26:591-599.

24. Hirko AC, R Dallasen, S Jomura and Y Xu. (2008). Modulation of inflammatory responses after global ischemia by transplanted umbilical cord matrix stem cells. Stem Cells 26:2893-2901.

25. Mitchell KE, ML Weiss, BM Mitchell, P Martin, D Davis, L Morales, B Helwig, M Beerenstrauch, K Abou-Easa, T Hildreth, D Troyer and S Medicetty. (2003). Matrix Cells from Wharton's Jelly form neurons and glia. Stem Cells 21:50-60. 
26. Wislet-Gendebien S, F Bruyère, G Hans, P Leprince, G Moonen and B Rogister. (2004). Nestin-positive mesenchymal stem cells favour the astroglial lineage in neural progenitors and stem cells by releasing active BMP4. BMC Neurosci 5:33-44.

27. Chen Q, Y Long, X Yuan, L Zou, J Sun, S Chen, JR Perez-Rolo and K Yang. (2005). Protective effects of bone marrow stromal cell transplantation in injured rodent brain: synthesis of neurotrophic factors. J Neurosci Res 80:611-619.

28. Munoz RM, BR Stoutenger, AP Robinson, JL Spees and DJ Prockop. (2005). Human stem/progenitor cells from bone marrow promote neurogenesis of endogenous neural stem cells in the hippocampus of mice. PNAS 102:18171-18176.

29. Crigler L, RC Robey, A Asawachaicharn, D Gaupp and DG Phinney. (2006). Human mesenchymal stem cell subpopulations express a variety of neuro-regulatory molecules and promote neuronal survival and neuritogenesis. Exp Neurol 198:54-64.

30. Sarugaser R, D Lickorish, D Baksh, MM Hosseini and JE Davies. (2005). Human umbilical cord perivascular cells (HUCPV) cells: a source of mesenchymal progenitors. Stem Cells 23:220-229.

31. Baksh D, R Yao and RS Tuan. (2007). Comparison of proliferative and multilineage differentiation potential of human mesenchymal stem cells derived from umbilical cord and bone marrow. Stem Cells 25:1384-1392.

32. Lu J, Y Wu, N Sousa and OF Almeida. (2005). SMAD pathway mediation of BDNF and TGF beta 2 regulation of proliferation and differentiation of hippocampal granule neurons. Development 132:3231-3242.

33. Giulian D and T Baker. (1986). Characterization of ameboid microglia isolated from developing mammalian brain. J Neurosci 6:2163-2178.
34. Cory AH, TC Owen, JA Barltrop and JG Cory. (1991). Use of an aqueous soluble tetrazolium/formazan assay for cell growth assays in culture. Cancer Commun 3:207-212.

35. Salih V, K Franks, M James, GW Hastings and JC Knowles. (2000). Development of soluble glasses for biomedical use Part II: The biological response of human osteoblast cell lines to phosphatebased soluble glasses. J Mater Sci Mater Med 11:615-620.

36. Silva GA, FJ Costa, OP Coutinho, S Radin, P Ducheyne and RL Reis. (2003). In vitro degradation and cytocompatibility evaluation of novel soy and sodium caseinate-based membrane biomaterials. J Mater Sci Mater Med 14:1055-1066.

37. Ennis J, C Götherström, K Le Blanc and JE Davies. (2008). In vitro immunologic properties of human umbilical cord perivascular cells. Cytotherapy 10:174-181.

Address correspondence to: Prof. Nuno Sousa

Life and Health Sciences Research Institute (ICVS) School of Health Sciences University of Minho Campus de Gualtar Braga 4710-057

Portugal

E-mail: njcsousa@ecsaude.uminho.pt

Received for publication July 29, 2009

Accepted after revision August 25, 2009

Prepublished on Liebert Instant Online August 25, 2009 